\title{
NEAR INFRARED IMAGING MAGNETOMETRY
}

\author{
DOUGLAS RABIN \\ National Solar Observatory, National Optical Astronomy Observatories, ${ }^{*}$ \\ P. O. Box 26732, Tucson, Arizona 85726, U.S.A.
}

\begin{abstract}
Infrared array detectors are a new and promising tool for investigating the properties of magnetic field concentrations in the solar photosphere. Array measurements provide large statistical samples of polarized line profiles and display the spatial organization of the magnetic field. The wavelength region near $1.6 \mu \mathrm{m}$ has important advantages for magnetometry: spectral lines with magnetic sensitivites ranging from low to very high; low continuum opacity; high continuum flux; and the possibility of sub-arcsecond angular resolution with existing telescopes. Initial results have extended earlier work on the distribution of field strength and flux in plages and revealed new properties specifically connected with spatial structure. The quality and flexibility of near infrared magnetographs can be expected to improve rapidly.
\end{abstract}

Key words: flux tubes - infrared: stars - instrumentation: polarimeters - Sun: faculae, plages Sun: magnetic fields

\section{Rationale}

The 1-2.5 $\mu \mathrm{m}$ spectral region offers scientific and technical advantages for the measurement of photospheric magnetic fields. As discussed more fully elsewhere in this volume (Solanki, 1993), the ratio of Zeeman line splitting to non-magnetic line width varies approximately as $g \lambda$, where the Landé factor can attain $g=3$ both in the visible and the near infrared. Also, lines in the continuum opacity window near $1.6 \mu \mathrm{m}$ are formed deep in the photosphere - where the magnetic field is strong and usually close to LTE. Contamination by stray light is smaller than at visible wavelengths, both because the continuum intensity is less sensitive to temperature and because there is typically less instrumental and atmospheric scattering; the relative freedom from stray light is particularly important in sunspots.

Here I shall address the specific rationale for imaging magnetometry in the near infrared. From the most general perspective, images with angular resolution $\sim 1^{\prime \prime}$ are in many ways the common language of observational solar physics. An image of this kind can be easily registered and compared to a wide variety of other images which measure various quantities and are often obtained on a routine basis. By contrast, a single-point measurement, particularly if it has crude angular resolution, raises questions of location and context. To which patch of plage, for example, does the measurement refer? How different would the measurement be if it were taken $5^{\prime \prime}$ away? How many measurements, distributed over what area, are needed to obtain a fair statistical sample? Imaging measurements may not eliminate these questions, but they make them easier to address. One may speculate that the difficulty of obtaining images with available technology was largely responsible for the hiatus in solar observations following the pioneering work of Harvey and Hall (Harvey and Hall, 1975; Harvey, 1977), although single-detector instruments could be used to begin the stellar measurements reviewed elsewhere in this volume (Saar, 1993). Stenflo, Solanki, and Harvey (1987) were able to make important

* Operated by the Association of Universities for Research in Astronomy, Inc., under cooperative agreement with the National Science Foundation. 
progress in the solar case, despite the absence of imaging capability, by combining the extreme spectral resolution and accuracy of a Fourier transform spectrometer with full radiative transfer analysis.

If we grant the desirability of obtaining magnetic images, a strong case can be made for observing Fe I $6388.6 \mathrm{~cm}^{-1}(1.5649 \mu \mathrm{m})$. It has high magnetic sensitivity $(g=3)$ and is conveniently (for an array detector) near a line with a different Zeeman sensitivity (Fe I $6386.9 \mathrm{~cm}^{-1}, g_{\mathrm{eff}}=1.53$ ). It is instructive to compare from a technical perspective the $1.56 \mu \mathrm{m}$ lines with the emission line $\mathrm{Mg}$ I $12.32 \mu \mathrm{m}$ $(g=1)$ and favorable visible-wavelength lines such as Fe I 5250.2 $\AA(g=3)$ and $5247.1 \AA$; Solanki (1993, in these proceedings) makes a similar comparison from the viewpoint of line formation and profile fitting.

Angular Resolution. Magnetic flux tubes in plage and network areas are sub-arcsecond structures, with characteristic diameters of perhaps $200 \mathrm{~km}$ (Steiner, 1993). Resolving these structures will almost certainly require observations from space or adaptive optics on the ground. A lesser but still important goal is to study the properties of single flux tubes even when they are not resolved. For spaceborne observations, visible or ultraviolet wavelengths have an advantage because they can achieve resolutions well below $1^{\prime \prime}$ with telescopes of aperture $\lesssim 1 \mathrm{~m}$. On the ground, observations at $12 \mu \mathrm{m}$ are diffraction-limited to $2^{\prime \prime}$ resolution on the largest available (1.5-m) telescope, although, for the purpose of isolating flux tube properties, the great magnetic resolution of the $12-\mu \mathrm{m}$ lines is a compensating advantage (Deming et al., 1988). Visible-light observations should be able to achieve $\sim 0$ '! 2 resolution on a $75-\mathrm{cm}$ telescope using adaptive optics, although the technical problems are formidable, especially for polarimetry. Observations at $1.6 \mu \mathrm{m}$ offer an attractive middle ground: the diffraction limit is below 0.3 on a $1.5-\mathrm{m}$ telescope, and the adaptive optics needed to approach that limit are expected to be considerably less elaborate than at visible wavelengths (Roddier and Graves, 1993).

Photon Flux. Ground-based polarimetry is best done by rapidly modulating between complementary polarization states (e.g., Stokes $I \pm V$ ) in order to minimize the signal-to-noise degradation due to seeing. Extracting information on the intrinsic magnetic field strength and direction - rather than just magnetic flux - calls for high spectral resolution, $\lambda / \Delta \lambda \gtrsim 10^{5}$. These two objectives, combined with the impetus toward high angular resolution, mean that photon flux is an important consideration for imaging magnetometry. The photon flux per linewidth (and per square arcsecond) is actually slightly higher for Fe I $1.565 \mu \mathrm{m}$ than for Fe I $5250 \AA$; but the flux for $\mathrm{Mg} \mathrm{I} 12.32 \mu \mathrm{m}$ is lower by more than an order of magnitude.

Polarized Signal. The greatest barrier to vector magnetometry in the visible is the low levels of linear polarization $\left(10^{-3}-10^{-4}\right)$ produced by incomplete Zeeman splitting. At $1.6 \mu \mathrm{m}$, and even more so at $12 \mu \mathrm{m}$, the prevalence of strong splitting means that Stokes $Q$ and $U$ are comparable in strength to Stokes $V$. On the other hand, infrared lines are generally weaker, reducing the amplitude of all Stokes components; and, because all the components are comparably strong, linear-to-circular crosstalk cannot be ignored as it often can be in the visible (although, at $12 \mu \mathrm{m}$, it may be possible to ignore instrumental polarization altogether). 


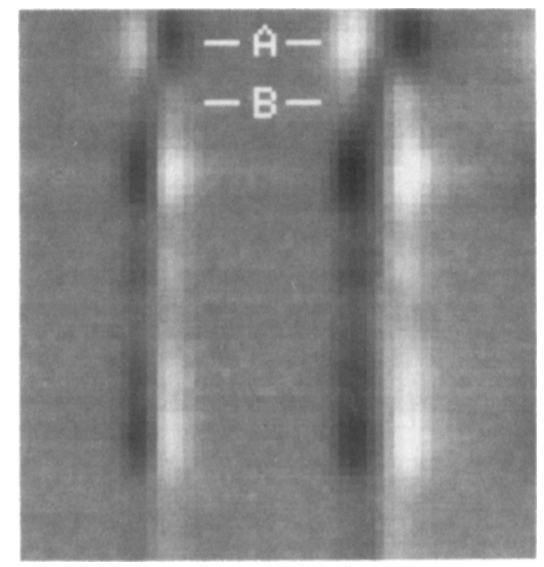

Fig. 1. Spatial-spectral Stokes $V$ image in a plage region. The spectral lines are Fe I $6388.6 \mathrm{~cm}^{-1}$ (right) and Fe I $6386.9 \mathrm{~cm}^{-1}$ (left). The spatial extent (top to bottom) is $53^{\prime \prime}$. The features marked $A$ and $B$ are discussed in the text.

Detectors. The standard detector for $0.3-1 \mu \mathrm{m}$ is the CCD, which has high quantum efficiency and is readily available in $2048 \times 2048$ format. Several infrared array detectors that operate at $1.56 \mu \mathrm{m}(\mathrm{HgCdTe}, \mathrm{InSb}, \mathrm{PtSi})$ are commercially available in formats ranging from $128 \times 128$ to $512 \times 512$. Recently, their formats have grown faster than CCD formats. A $512 \times 512$ format is already large enough to image an active region with good angular resolution. $\mathrm{HgCdTe}$ and $\mathrm{InSb}$ devices also have high quantum efficiency. At present, arrays for the 5-20 $\mu \mathrm{m}$ range are usually smaller in format and more expensive; but they too will enter a period of rapid growth. Many infrared arrays are designed to run at video or higher rates, which matches the need for rapid polarization modulation.

\section{Initial Results}

\subsection{Spectra}

Figure 1 is an example of a Stokes $V$ spatial-spectral image at $1.565 \mu \mathrm{m}$, obtained in a plage region using the NSO Near Infrared Magnetograph - NIM (Rabin, 1992b). In the vicinity of the inversion line of the line-of-sight field (located between the features marked $A$ and $B$ ), there are obvious changes in the splitting and central wavelength of the $\sigma$ components. Elsewhere in these proceedings, Solanki (1993) discusses the interpretation of these $V$ profiles in terms of a siphon flow in the fluxtube arches near the inversion line. However, simple Seares-profile fitting detects significant variations in field strength throughout this image and in other plage areas. Figure 2 illustrate fits to $V$ profiles and the formal uncertainties in the 


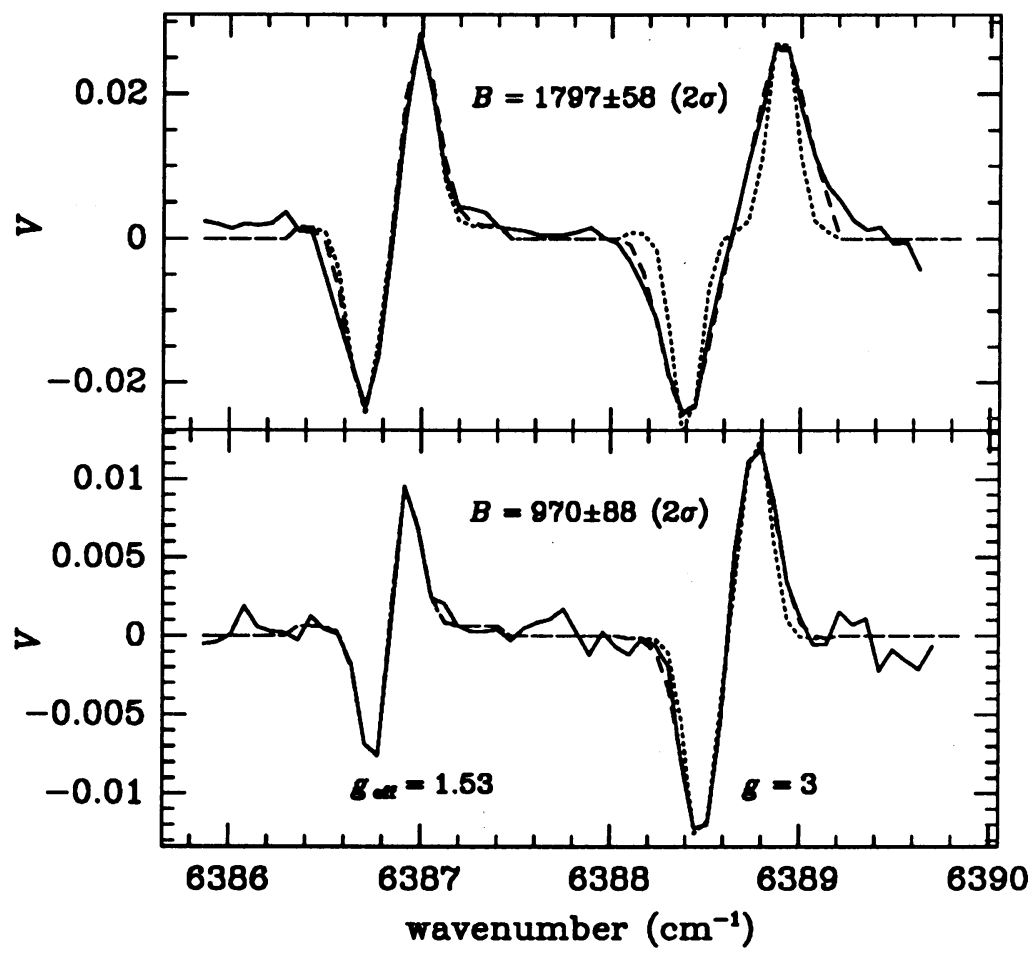

Fig. 2. Examples of observed and fitted $V$ profiles. Solid: observed. Dashed: fitted with the width of the sigma components as a free parameter. Dotted: fitted with the width of the sigma components fixed at the quiet-Sun line width.

determination of field strength. Figure 2 also illustrates a pervasive feature of the plage profiles: the $\sigma$ components are broader than what is obtained by simply splitting a quiet-Sun line profile. Zayer et al. (1989) demonstrated that the $\sigma$ broadening is magnetic in origin and that the degree of broadening is consistent with the vertical gradient of flux-tube field strength over the region of line formation.

\subsection{Magnetic MaP}

Figure 3 shows cospatial maps of magnetic flux and magnetic field strength in a plage region, derived from infrared spectra such as those shown in Figure 2. The bottom panel is a "true-field" magnetogram. The field-strength map shows that there are spatially coherent variations in $|\mathbf{B}|$ (at the level of line formation) throughout this plage region (Rabin, 1992a). Field strength and flux are related, but not one-to-one. For example, Region $\mathrm{A}$ includes the most intense flux in the field of view, but Regions B and C contain stronger magnetic fields, and patches of relatively strong field can occur in areas of weak flux (Region B). This relationship is explored further below.

Figure 3 can only indicate some of the potential of near-infrared magnetic map- 

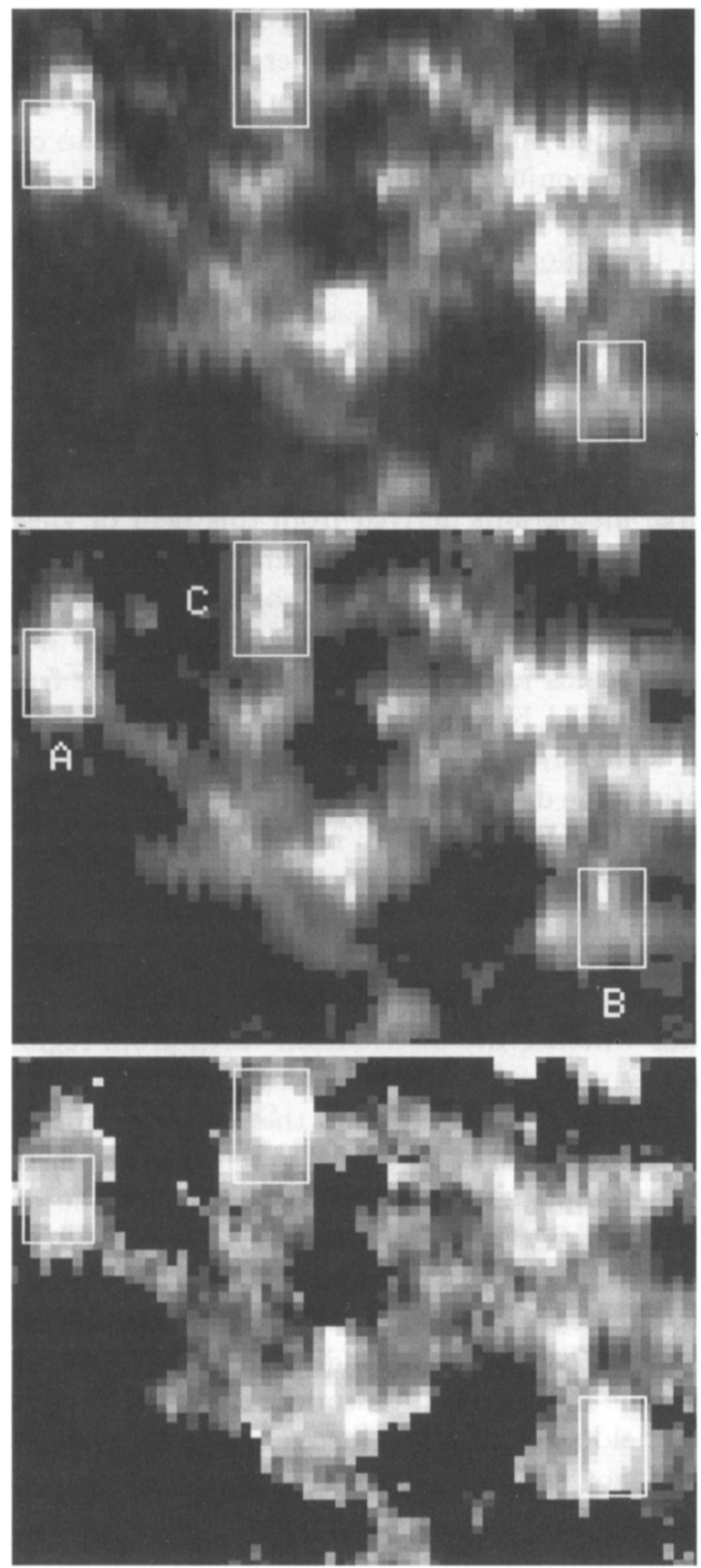

Fig. 3. Magnetic flux and magnetic field strength in a plage region. Top: model-independent magnetic flux, proportional to $\int|V| d \lambda / I_{c}$, where $I_{c}$ is the unpolarized continuum intensity. Middle: magnetic flux as measured by the amplitude of the fitted profile. The grayscale (black-to-white) is linearly mapped to the range $0.00-0.03$. Bottom: true magnetic field strength. The grayscale is linearly mapped to the range 1000-1600 Gauss. In the bottom two maps, pixels are blacked out where the profile did not satisfy the convergence and signal-to-noise criteria. The field of view is $74^{\prime \prime} \times 55^{\prime \prime}$. 
ping, which is in its infancy. Efforts are being directed toward creating vector-field maps from complete Stokes data. The Doppler information will be more fully utilized (McPherson, Lin, and Kuhn, 1992, give an example of a Doppler map around a sunspot derived from $1.565 \mu \mathrm{m}$ data). It should be possible to create spatial images of $\sigma$-broadening and, in conjunction with visible-light diagnostics, of the thermal properties of flux tubes. In sunspots, the relationship between magnetic and thermal properties can be explored from infrared data alone (Kopp and Rabin, 1993, in these proceedings).

\subsection{Statistical Properties}

Even though the angular field of Figure 3 is small, it provides a much larger statistical sample than can be obtained practicably with point-by-point measurements. Figure 4 shows three histograms derived from this field of view: field strength $B$, amplitude factor $A_{f}$, and $\Delta \nu_{E}$, the Gaussian line width of the fitted $\sigma$ components. $A_{f}$ is the fitted amplitude of the $V$ profile normalized by the maximum value it could achieve if the spectral line had its quiet-Sun central depth. To a first approximation, $A_{f}=f R \delta \cos \gamma$, where $f$ (filling factor) is the fraction of the spatial resolution element occupied by the flux tube, $\gamma$ is the inclination of the magnetic field to the line of sight, $\delta$ is the continuum contrast of the tube, and $R$ is the ratio of the absorption-line depths inside and outside the tube after the profiles are separately normalized to unit continuum intensity. Because both the lines and the continuum are relatively insensitive to temperature, variations in $\delta$ and $R$ are not expected to contribute much to the variation of $A_{f}$.

The width of the field-strength histogram is consistent with the range of plage field strengths measured by Harvey (1977). In future observations, it will be important to determine whether the distribution varies from region to region, and to explore the spatial environments of strong- and weak-field plages.

Figure 5 is a statistical representation of the relationship between field strength and flux (or $V$ amplitude) illustrated by the labelled boxes in Figure 3. The most striking feature is the empty region in the upper left of the diagram. The origin of this property is not known. One may speculate that flux tubes in highly filled areas are relatively cool, because the efficiency of convective transport is locally reduced, and therefore relatively transparent, because the $\mathrm{H}^{-}$opacity is lower. The line is formed deep in the atmosphere, leading to high measured field strengths. In sparsely filled areas, the individual tubes may be collected into cool clumps (again with high measured field strengths), or instead spread out more uniformly. Quasiisolated tubes would not suppress convective transport as effectively and would experience more radiative exchange with the surrounding atmosphere, resulting in higher temperature and pressure, increased opacity, and lower measured field strengths. Solanki suggests other possible interpretations based on a small sample of similar data (Solanki, 1993, Fig. 4).

\section{Prospects}

So far, near infrared imaging magnetometry has only scratched the surface of the scientific possibilities open to it. The following is a heterogeneous sample of expected 

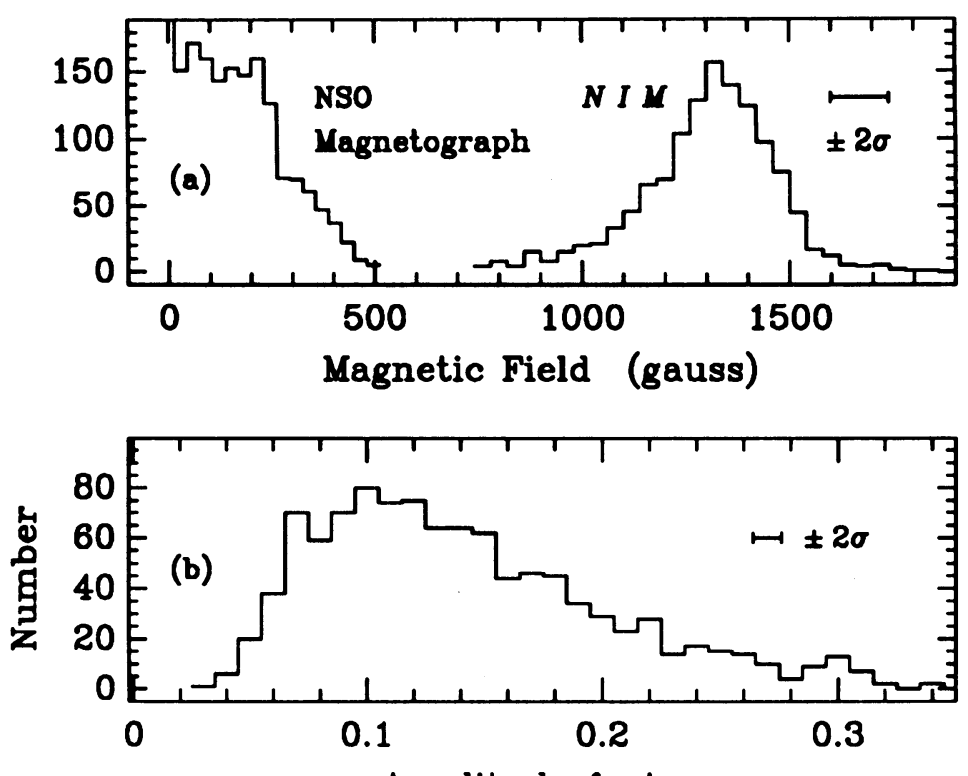

Amplitude factor

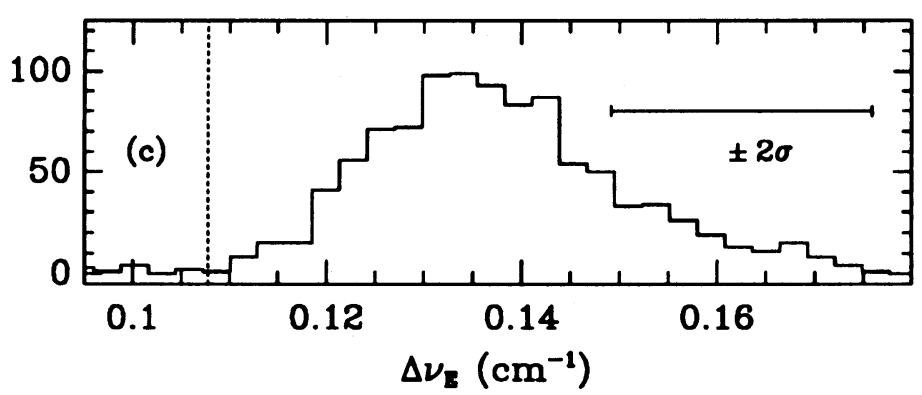

Fig. 4. Histograms of $(a)$ true field strength, measured by NIM, compared to apparent field strength, measured by the NSO synoptic magnetograph; $(b) A_{f}$, the normalized amplitude of the $V$ profile; $(c)$ width of the sigma components. The dotted line in Panel $\mathrm{c}$ is the width of the line in the quiet Sun. All error bars indicate $\pm 2 \sigma$ uncertainties in the individual measurements that contribute to the bins.

observational advances and what might be learned from them.

1. Spectral lines in the visible are often more temperature-sensitive than nearinfrared atomic lines; the $1.56 \mu \mathrm{m}$ lines offer a more sensitive determination of field strength at the level they are formed. Thus, simultaneous observations at $1.56 \mu \mathrm{m}$ and in the visible should be a powerful probe of the flux-tube atmosphere.

2. Now that the formation of the $12 \mu \mathrm{m}$ lines is well understood (Rutten and Carlsson, 1993; Avrett, Chang, and Loeser, 1993), simultaneous observations at 


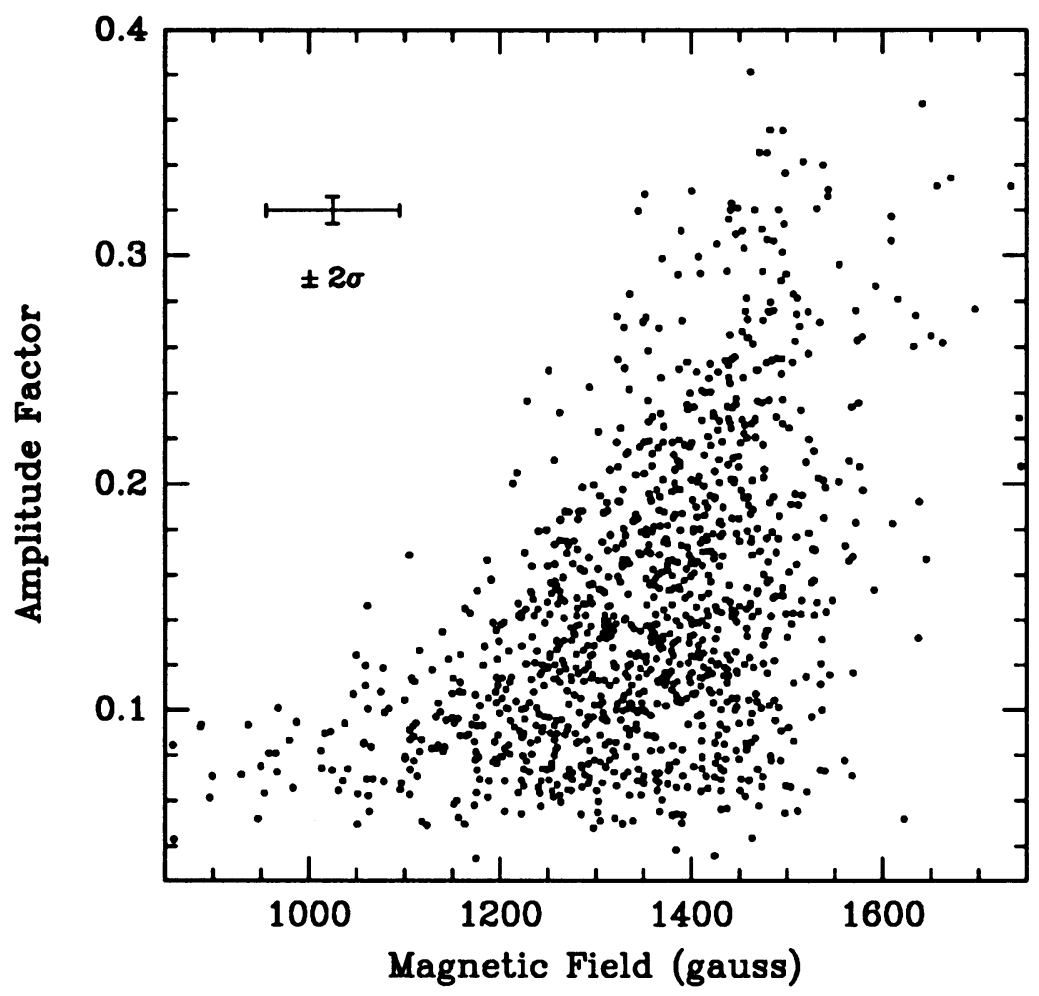

Fig. 5. Scatter plot of field strength versus $A_{f}$, the Stokes $V$ amplitude factor. A typical $\pm 2 \sigma$ uncertainty of fit along each axis is shown.

$1.6 \mu \mathrm{m}$ and $12 \mu \mathrm{m}$ should allow an unambiguous measurement of field strength differences (flux-tube spreading) over the vertical span of the photosphere.

3. Complete Stokes polarimetry - i.e., vector magnetometry - is, in the infrared, a relatively straightforward analogue of the circular polarimetry discussed above. Comparing $1.6 \mu \mathrm{m}$ observations with visible-light data from, e.g., the HAONSO Advanced Stokes Polarimeter (Lites et al., 1991) will be particularly valuable in the periphery of sunspots, where the field geometry changes rapidly with height.

4. Buoyancy tends to drive an isolated flux tube to a vertical orientation in the atmosphere (Spruit, 1981). However, flux tubes are always, to some degree, part of a larger magnetic-field configuration. Near an inversion line, for example, we should find substantial inclinations. Because of the comparable magnitude of all the Stokes components, $1.56 \mu \mathrm{m}$ observations in plage regions should provide relatively direct information about the inclination of flux tubes.

5. As mentioned earlier, Doppler information from infrared lines has barely been exploited. In sunspots, the infrared data will probe flow conditions deep in the photosphere. In plage flux tubes, the occurrence of siphon flows needs to be 
confirmed and buttressed with time series, since such flows must be transient.

6. Bigger and better array detectors will help in many areas. It will no longer be necessary to compromise spectral resolution in order to include a usable wavelength range on the array. Lower dark current and readout noise will increase the polarization sensitivity, eventually to the point where we can study the strength of quiet network and internetwork fields.

7. NIM employs a nematic liquid-crystal optic as a voltage-controlled variable retarder for measuring the Stokes vectors. The present device requires as much as $20 \mathrm{~ms}$ to change state and stabilize, during which time a full data frame is contaminated and therefore discarded. A faster cadence is desirable to supress the noise introduced by rapid seeing variations. It is likely that both ferroelectric and nematic liquid-crystal retarders will soon be capable of good performance in the infrared at video rates.

8. NIM scans the slit of a large spectrograph across the solar image. Just as there are spectrograph- and filter-based magnetographs in the visible, each with distinct advantages, it makes sense to develop a filter-based infrared magnetograph. It will have a faster cadence, suitable for studies related to solar activity, and it will be adaptable to balloon- or spaceborne telescopes.

\section{Acknowledgements}

I am grateful to the NIM team - D. Jaksha, G. Kopp, C. Mahaffey, C. Plymate, and J. Wagner - for helping to make the concept a reality. This work was assisted by the NASA Supporting Research and Technology program in solar physics.

\section{References}

Avrett, E. H., Chang, E. S., and Loeser, R.: 1993, these proceedings.

Deming, D., Boyle, R.J., Jennings, D.E., and Wiedemann, G.: 1988, Astrophys. J. 333, 978.

Harvey, J. W.: 1977, in E. A. Müller (ed.), Highlights of Astronomy 4, 223.

Harvey, J. W., and Hall, D.: 1975, Bull. Amer. Astron. Soc. 7, 459.

Kopp, G., and Rabin, D.: 1993, these proceedings.

Lites, B. W., Elmore, D., Murphy, G., Skumanich, A., Tomczyk, S., and Dunn, R. B.: 1991, in L. November (ed.), Solar Polarimetry, Proc. 11th Sacramento Peak Workshop, NSO, Sunspot, New Mexico, p. 3.

McPherson, M. R., Lin, H., and Kuhn, J. R.: 1992, Solar Phys. 139, 255.

Rabin, D.: 1992a, Astrophys. J. (Letters) 390, L103.

Rabin, D.: 1992b, Astrophys. J. 391, 832.

Roddier, F., and Graves, J. E.: 1993, these proceedings.

Rüedi, I., Solanki, S. K., and Rabin, D. 1992, Astron. Astrophys. 261, L21.

Rutten, R. J., and Carlsson, M.: 1993, these proceedings.

Saar, S.: 1993, these proceedings.

Solanki, S. K.: 1993, these proceedings.

Spruit, H.: 1981, in S. Jordan (ed.), The Sun as a Star, NASA SP-450, NASA, Washington, D.C., p. 385 .

Steiner, O.: 1993, these proceedings.

Stenflo, J. O., Solanki, S. K., and Harvey, J. W.: 1987, Astron. Astrophys. 173, 167.

Zayer, I., Solanki, S. K., and Stenflo, J. O.: 1989, Astron. Astrophys. 211, 463. 